\section{FRI0201 ETANERCEPT RETENTION PATTERNS AND FACTORS ASSOCIATED WITH TREATMENT DISCONTINUATION: A RETROSPECTIVE COHORT STUDY USING CANADIAN CLAIMS-LEVEL DATA}

M. Khraishi ${ }^{1}$, Y. Zhang ${ }^{2}$, J. Ivanovic ${ }^{2}$, B. Millson ${ }^{2}$, M.-J. Brabant ${ }^{2}$, K. Charland ${ }^{2}$ E. Singh ${ }^{3}$, J. Woolcott ${ }^{4}, \mathrm{H}$. Jones ${ }^{3}{ }^{1}{ }^{1}$ Faculty of Medicine, Memorial University of Newfoundland, St. Johns: ${ }^{2}$ Health Access \& Outcomes, QuintilesIMS, Kanata, Canada; ${ }^{3}$ Inflammation and Immunology, Global Medical Affairs, Pfizer Inc. Collegeville, United States; ${ }^{4}$ Health Economics and Outcomes Research, Pfizer Inc., Kirkland, Canada

Background: Etanercept is a soluble TNF receptor (humanized protein) indicated for the treatment of immune-mediated inflammatory diseases such as rheumatoid arthritis (RA), psoriatic arthritis (PsA), ankylosing spondylitis (AS), and psoriasis (PsO). Limited information exists on the factors associated with long-term retention and use of etanercept in Canada in a real-world setting.

Objectives: To evaluate the 6-year retention rates of etanercept patients in Canada, and to identify factors associated with discontinuation.

Methods: A retrospective cohort study was conducted using longitudinal prescription drug claims data from QuintilesIMS Private Drug Plan database (PDP), Ontario Public Drug Plan database (OPDP), and Quebec Public Drug Plan database (RAMQ). Between 07/2008 and 06/2010, bio-naïve patients who initiated etanercept were identified and followed for 72 months. 12-month retention rates were evaluated in 1-year increments for all patients retained on therapy at years $1,2,3,4$ and 5 and compared to retention rates in the first year. The covariates associated with time to discontinuation over the entire 72 month period were identified using a Cox proportional hazards regression model.

Results: The study identified 4,528 etanercept patients (61\% female, $85 \%$ rheumatic diseases, and $15 \% \mathrm{PsO}$ ) across Canada who started their therapy during the selection period. Overall, 12-month retention rates on etanercept increased significantly for patients following their first year on therapy $(p<.0001)$, with $66 \%$ of patients retained at year 1 vs. 12 - month retention rates of $79 \%$, $82 \%, 84 \%, 83 \%$ and $79 \%$ at year $2,3,4,5$ and 6 , respectively. A total of $17.1 \%$ $(n=771)$ of patients were retained for the entire 72 month study. Regression analysis showed PsO patients were less likely to be retained on therapy than other indications (HR 1.199; $p<.0001)$, older patients $(65+)$ were more likely to be retained than younger patients (HR $0.802 ; p<.0001$ ), and public plan patients (ODB HR 0.735 , RAMQ HR $0.55 ; p<.0001$ ) were more likely to be retained than private plan patients.

Conclusions: Etanercept patient retention likelihood increased the more years a patient was retained on therapy. This pattern was consistent across therapeutic areas, sex, age, and payers. Age, indication, and payer were found to have a significant impact in determining etanercept patients' time to therapy discontinuation. With better understanding of factors associated with retention, patient support programs can be designed to address the specific needs of at-risk groups while supporting patients stable on therapy.

Disclosure of Interest: M. Khraishi Consultant for: Pfizer Canada and Amgen Canada, Y. Zhang: None declared, J. Ivanovic: None declared, B. Millson: None declared, M.-J. Brabant: None declared, K. Charland: None declared, E. Singh: None declared, J. Woolcott: None declared, H. Jones: None declared DOI: 10.1136/annrheumdis-2017-eular.6312

\section{FRI0202 THE DIFFERENT EFFECT OF TNF $\alpha$ BLOCKERS AND SDMARDS ON LIPOPROTEIN SUBCLASSES IN RA PATIENTS. A PERSPECTIVE CONTROLLED LONGITUDINAL STUDY}

M. Zakalka ${ }^{1}$, M. Karakota ${ }^{2}$, G. Koliakos ${ }^{3}$, L. Settas ${ }^{1} \cdot{ }^{1}$ Division of Clinical Immunology/Rheumatology, 1st Internal Medicine Department, AHEPA University Hospital, Medical School, Aristotle University; ${ }^{2}$ Laboratory of Biological Chemistry; ${ }^{3}$ Laboratory of Biological Chemistry, Aristotle University, Thessaloniki, Greece

Background: The different antirheumatic drugs reduce inflammation in RA patients, causing alterations in cholesterol levels (mainly decreasing LDL and increasing HDL cholesterol levels), but HDL and LDL structure and function predict cardiovascular disease better than LDL and HDL cholesterol levels.

Objectives: The investigation of the qualitative changes of LDL and HDL lipoprotein subclasses in RA patients who are good responders, depending on the anti-inflammatory treatment (sDMARDs vs anti-TNFa+sDMARDs) and their in between associations.

Methods: 85 patients ((89\%) 76females) with established RA (mean disease duration $\geq 5 \mathrm{yrs}$ ), mean age $57 \mathrm{yrs}$ (SD: 12yrs), without known cardiovascular disease, D/M and thyroid disorders, on SDMARD (MTX, LEF, SSZ, low dose prednisolone or combination) and naïve to biologic treatment, were divided into two groups: the 1st one of 43 patients who had $\mathrm{DAS}_{28}>3.2$ and were given in addition a TNF $\alpha$ inhibitor (23 patients had golimumab and 20 patients had certolizumab-pegol) for at least 54weeks (mean treatment duration 18 months) with good clinical response according to Eular and the 2nd group of 42 patients (disease control group) with $\mathrm{DAS}_{28} \leq 3.2$, who continued on sDMARDs and followed closely the same time period so as to ensure, as well, good clinical response without biologic therapy. Plasma electrophoresis was performed in two time points, before and after anti-TNF $\alpha$ administration and on the same time for the control group, with non-denaturing polyacrylamide gel (ND-PAGE) for size-based separation of lipoprotein subclasses, a standard laboratory technique that indentify various HDL subspecies separable on the basis of average diameter/size into 6 distinct subclasses (HDL-1, HDL2a, HDL2b, HDL3a, HDL3b, HDL3c), as well as LDL lipoprotein into 2 subfractions (LDL-B, LDL-A.)

Results: The RA patients on DMARDs had a percent reduction of LDL-B subfraction by $11.9 \%(p=0.014)$, but a significant increase of HDL-3c subclass by $2.38 \%(p=0.049)$. In a multivariate model of stepwise logistic regression the after (treatment)-LDL-B subfraction in these patients was found to have significant positive association only with pro-LDL-B subfraction (odds ratio: 10.95, $\% \mathrm{Cl}(1.59-62.79))$. In the RA patients who took TNF $\alpha$ inhibitors was observed a prominent percent decrease of the more lipoprotein subclasses: LDL-B by $2.33 \%(p=0.005)$, LDL-A by $2.33 \%(p=0.0001)$, HDL3a by $11.63 \% \quad(p=0.072)$ and HDLc by $4.65 \%(p=0.035)$. Accordingly, in stepwise logistic regression the after-(treatment) LDL-B subfraction had a significant positive association only with pro-LDL-B subfraction (odds ratio: $12.91,95 \% \mathrm{Cl}(2.2-75.83)$ ), as well as, after-LDL-A with pro-LDL-A subfraction (odds ratio:36.16, 95\% Cl (5.05-258.89)), whereas the reduced after (treatment)-HDL3c subclass was significantly positively associated with pro-HDL3a subclass (odds ratio:8.15, 95\% $\mathrm{Cl}(1.04-63.65)$ ) and after-HDLa subclass (odds ratio:22.09, $95 \% \mathrm{Cl}(3.56-137.18)$ ).

Conclusions: The discrepancy of qualitative modification of lipoprotein subclasses (LDL and HDL) after treatment with different antirheumatic drugs (sDMARDs, anti-TNFa+sDMARDs) demonstrate their different effect on RA dyslipidemia and their subsequent antiatherogenic prospective.

Disclosure of Interest: None declared

DOI: 10.1136/annrheumdis-2017-eular.4507

\section{FRI0203 GOLIMUMAB IN BIOLOGIC-NAIIVE PATIENTS WITH ESTABLISHED RHEUMATOID ARTHRITIS (RA), PSORIATIC ARTHRITIS (PSA) OR ANKYLOSING SPONDYLITIS (AS) - SUBANALYSIS FROM THE NON-INTERVENTIONAL EVALUATION GO-NICE}

K. Krüger ${ }^{1}$, G.R. Burmester ${ }^{2}$, S. Wassenberg ${ }^{3}$, M. Bohl-Bühler ${ }^{4}$, M.H. Thomas ${ }^{5} .{ }^{1}$ Rheumatologisches Praxiszentrum, München; ${ }^{2}$ Department of Rheumatology and Clinical Immunology, Charité-Universitätsmedizin, Berlin ${ }^{3}$ Rheumazentrum Ratingen, Ratingen; ${ }^{4}$ Rheumahaus Potsdam GbR, Potsdam; ${ }^{5}$ Medical Affairs, MSD Sharp \& Dohme GmbH, Haar, Germany

Background: Golimumab (GLM) has demonstrated efficacy and safety in several randomized clinical trials with biologic-naïve patients. However, data from effectiveness and patient-reported outcomes (PROs) parameters in daily clinical practice in Germany are still lacking

Objectives: The aim of this subanalysis is to assess Golimumab on the effectiveness, and PROs in biologic-naïve patients with established RA, PsA or AS

Methods: This is a subanalysis of the non-interventional, prospective, 24-month study GO-NICE. Biologic-naïve patients with established RA, PsA or AS starting with GLM 50mg SC once monthly in a real life setting in Germany. Endpoint measures: disease activity DAS28, PsARC and BASDAI. PROs included QoL (EQ-5D-3L), functionality $(\mathrm{FFbH})$, fatigue (FACIT-F). Safety data were also collected

Results: RA patients ( $\mathrm{n}=265)$ : Mean age $54.5 \mathrm{yr}, 82.1 \%$ of the patients were female, $77.3 \%$ ( $n=204)$ were rheumatoid factor (RF) positive, and $76.4 \%(n=201)$ had anti-ccp antibodies at BL.

The DAS28 score at BL was 5.0 and dropped significantly to 2.9 within 24 months $(\mathrm{p}<0.0001 \mathrm{v}$. BL). After 3 months of treatment, $45.2 \%$ of patients had LDA (DAS28 $\leq 3.2$ ), which increased to $50.8 \%$ after 6 month and $64.9 \%$ after 24 months.

PSA patients $(\mathrm{n}=247)$ : Mean age $49.7 \mathrm{yr}, 53.8 \%$ of the patients were female, $42.1 \%(n=104)$ had a nail involvement, $25.5 \%(n=63)$ dactylitis and $13.8 \%(n=34)$ enthesitis at BL. The proportion of patients achieving a response (mod PsARC) was $64 \%, 72.2 \%$ and $77.7 \%$ at 3,6 and 24 months, respectively.

AS patients ( $n=246)$ : Mean age $41.9 \mathrm{yr}, 70.7 \%$ of the patients were male, $80.5 \%$ $(n=198)$ were HLA-B27 positiv. Most common extraarticular manifestations were: enthesitis (12.6\%), iritis (12.2\%), IBD (3.7\%), and dactylitis (2.8\%) at $\mathrm{BL}$.

The BASDAI at BL was 5.0 and dropped significantly to 2.0 within 24 months $(p<0.0001 \mathrm{vs}$. BL). The proportion of patients achieving a response (BASDAI 50) was $62.2 \%, 66.9 \%$ and $76.9 \%$ at 3,6 and 24 months, respectively.

An improvement of quality of life (QoL) by EQ-5D-3L was seen after 6 months and was maintained over 24 months. The patients' health state today (EQ VAS) improved from 52.3 at $\mathrm{BL}$ to $64.9(\mathrm{RA})$, from 49.0 to 66.3 (PsA) and from 49.2 to 70.6 (AS). The functional ability (FFbH) improved significantly $(p<0.0001$ vs. BL) from 73.1 to 80.4 points (RA), from 73.0 to 82.2 (PsA) and from 72.8 to 81.2 (AS). The mean Fatigue score (FACIT-F) increased from BL: 33.3 to 39.5 points (RA), from 31.6 to 38.4 points (PsA), and from 31.6 to 40.2 points (AS) (each $p<0.001$ vs $\mathrm{BL}$ ) within 24 months.

No new safety signals were detected.

Conclusions: GLM SC once-monthly showed after 3 months remarkable improvements in clinical effectiveness, patient-reported quality of life, functionality, and fatigue parameters and were maintained over 24 months in biologic-naive patients with established RA, PsA or AS.

At month $24,64.9 \%$ of RA patients achieved LDA status, $77.7 \%$ of PsA achieved positive PsARC response and $76.9 \%$ of AS patients achieved BASDAI 50. No new safety signals were detected. 
Disclosure of Interest: K. Krüger Consultant for: AbbVie, BMS, Celgene, Janssen Biologics, MSD, Pfizer, Roche, and Sanofi-Aventis, G. Burmester Consultant for: AbbVie, BMS, MSD, Pfizer, Roche, and UCB, S. Wassenberg Consultant for: AbbVie, Chugai, Janssen Biologics, MSD, Novartis, Pfizer, Roche, and UCB, M. Bohl-Bühler Consultant for: AbbVie, Hexal, MSD, Roche, and UCB, M. Thomas Employee of: MSD Sharp \& Dohme GmbH Germany

DOI: 10.1136/annrheumdis-2017-eular.5154

\section{FRI0204 COMPARISON THE LONG-TERM CLINICAL OUTCOMES BETWEEN NONTNF-INHIBITORS VERSUS TNF-I IN RA PATIENTS WHO FAILED TO A FIRST TNF-I}

P. Bogas ${ }^{1}$, C. Plasencia ${ }^{1}$, D. Pascual-Salcedo ${ }^{2}$, G. Bonilla ${ }^{1}$, E. Moral ${ }^{1}$, C. Tornero ${ }^{1}$, L. Nuño ${ }^{1}$, A. Villalba ${ }^{1}$, D. Peiteado ${ }^{1}$, A. Martinez ${ }^{2}$, B. Hernandez ${ }^{2}$, A. Balsa ${ }^{1} .{ }^{1}$ Rheumatology; ${ }^{2}$ Immunology, Hospital Universitario la Paz, Madrid, Spain

Background: There are many biological therapies for Rheumatoid Arthritis (RA) with different mechanisms of action and good efficacy rate; however, up to $40 \%$ of patients (pts) fail to respond to the 1st biologic agent, and it is still not clear what strategy to follow after showing inadequate response to tumor necrosis factor $\alpha$ inhibitors (TNF-i)

Objectives: To assess the clinical response and survival (SVV), in our cohort of RA pts that discontinued the 1st TNF-i, of a 2nd TNF-i vs a nonTNF-i, both in the global cohort and in the subpopulation that dropped out the 1st TNF-I due to inefficacy

Methods: This observational study included 110 pts in the RA-Paz cohort who previously suspended Ifx (68\%) or Ada (32\%) between 1999-2016. Two groups were established as they switched to a TNF-i or nonTNF-i. Clinical response was evaluated by DAS28, Delta-DAS28 ( $\triangle \mathrm{DAS} 28$ ) and EULAR response (E-resp). The assessments were performed at $6(\mathrm{v}-6)$ and 12 months $(\mathrm{v}-12)$ since initiating 2nd biological agent and during the last visit prior to drug discontinuation or ending of the study for those who did not interrupt the drug (v-end). Statistical analysis was performed using SPSS version 20.0

Results: Of the 110 pts who had stopped Ifx or Ada as 1 st TNF-i, $65 \%$ changed to a 2 nd TNF-i. The $84 \%$ of the overall pts were women. The mean age was $64 \pm 14$ years and the mean time of 2 nd biologic drug was $3.71 \pm 3.51$ years. $61 \%$ associated methotrexate at the beginning of 2 nd biologic agent and $56 \%$ at the v-end, without differences between those who switched to TNF-i and those who did to nonTNF-i. At $v-6$ and $v-12$, there was no difference in $\triangle$ DAS28 [at $v-6: 1.3 \pm 1.4$ in TNF-i and $1.2 \pm 1.2$ in nonTNF-i $(\mathrm{p}=0.919)$, at $v-12: 1.3 \pm 1.5$ in TNF-I and $1.2 \pm 1.1$ in nonTNF-i $(\mathrm{p}=0.852)]$. In contrast, at $\mathrm{v}$-end, pts with nonTNF-i showed a higher clinical improvement ( $\triangle \mathrm{DAS} 28: 0.68 \pm 1.7$ in TNF-i, $1.8 \pm 1.1$ in nonTNF-i, $\mathrm{p}=0.002$ ). At $\mathrm{v}-6$, the TNF-i group achieved higher good E-resp rate ( $41 \%$ vs $18 \%, p=0.035)$, but there was no difference at $v-12(36 \%$ in TNF-I vs $23 \%$ in nonTNF-i, $p=0.435$ ). However, at v-end, the nonTNF-I group achieved better E-resp (good resp: $38 \%$ in nonTNF-i vs $25 \%$ in TNF-I, no resp $18 \%$ in nonTNF-i vs $50 \%$ in TNF-i, $p=0.01)$. Likewise, $100 \%(n=7)$ of the pts that finished 2nd biologic agent by remission, had changed to a nonTNF-i $(p<0.00001)$. There were no differences regarding 2nd biologic drug SVV (mean SVV time of $5.7 \pm 0.66$ in TNF-I, $4.3 \pm 0.59$ in nonTNF-i, $p=0.797$ ). When analyzing the cohort that discontinued 1st TNF-I because of inefficacy, at $v-6$ and $v-12$ there were no differences between switchers to TNF-I and nonTNF-i in $\triangle \mathrm{DAS} 28$ [v-6: $1.4 \pm 1.4$ vs $0.9 \pm 1 \mathrm{p}=0.164) ; \mathrm{v}-12: 1.5 \pm 1.4$ vs $1 \pm 1, \mathrm{p}=0.192)$ ], but at $\mathrm{v}$-end, the nonTNF-i group reached a higher $\triangle \mathrm{DAS} 28(0.9 \pm 1.5$ in TNF-i, $1.6 \pm 1$ in nonTNF-i, $p=0.031)$

\begin{tabular}{|c|c|}
\hline \multicolumn{2}{|c|}{ Demographic characteristics. } \\
\hline Age (years) & $64 \pm 13,9$ \\
\hline Sex (female) & $92(84 \%)$ \\
\hline Smokers. & $18(16 \%)$ \\
\hline BMI & $26,6 \pm 7,7$ \\
\hline Disease duration (xears) & $16,7 \pm 8,04$ \\
\hline RF + & $94(85 \%)$ \\
\hline Anti-CCP + & $96(89 \%)$ \\
\hline $\begin{array}{l}\text { Duration of treatment 1st } \\
\text { biological (years) }\end{array}$ & $3,2 \pm 3,1$ \\
\hline Basal DMARDs ( $2^{\circ}$ biological) & $99(90 \%)$ \\
\hline Basal MTX $\left(2^{\circ}\right.$ biological $)$ & $67(61 \%)$ \\
\hline v-Final FAMEs $\left(2^{\circ}\right.$ biological $)$ & $93(86 \%)$ \\
\hline v-Final MTX $\left(2^{\circ}\right.$ biological) & $60(56 \%)$ \\
\hline Basal CPR (2॰ biological) & $15,2 \pm 18,7$ \\
\hline Basal ESR $\left(2^{\circ}\right.$ biologicall $)$ & $42 \pm 24,1$ \\
\hline Basal DAS $\left(2^{\circ}\right.$ biological) & $5,55 \pm 1,3$ \\
\hline
\end{tabular}

Conclusions: In our sample of RA patients who suspended Ifx/Ada as 1st TNF-i, switching to a 2nd biologic agent did not show relevant clinical differences between a TNF-i and a nonTNF-i within the 1st year of treatment. However, in the long-term, switching to a nonTNF-i shows enhanced clinical benefits with no impact on survival vis-à-vis a 2nd TNF-i. Despite the efficacy of TNF-i, new therapeutic targets are needed for those who fail to respond to these biological agents

Disclosure of Interest: None declared

DOI: 10.1136/annrheumdis-2017-eular.4383

\section{FRI0205 CORRELATION OF PATIENT PREFERENCES TO TREATMENT OUTCOMES IN PATIENTS WITH RHEUMATOID ARTHRITIS (RA) TREATED WITH ANTI-TNF AGENTS IN GREECE. THE PANORAMA STUDY}

P. Sidiropoulos ${ }^{1}$, A. Bounas ${ }^{2}$, P. Athanassiou ${ }^{3}$, A. Andrianakos ${ }^{4}$, E. Petrikkou ${ }^{5}$, A. Drosos ${ }^{6}$, D. Vassilopoulos ${ }^{7}{ }^{1}$ Department of Rheumatology, Clinical Immunology and Allergy, University of Crete, Heraklion: ${ }^{2}$ Private practice, Patras; ${ }^{3}$ Rheumatology Division, General Hospital of Thessaloniki St. Paul, Thessaloniki; ${ }^{4}$ IASO General Hospital; ${ }^{5}$ Medical Affairs, MSD, Athens; ${ }^{6}$ Rheumatology Clinic, University of loannina, loannina: ${ }^{7}$ Joint Rheumatology Program, Clinical Immunology-Rheumatology Unit, 2nd Department of Medicine and Laboratory, National and Kapodistrian University of Athens, Athens, Greece

Background: Route and frequency of administration of treatment options may be an important differentiator between drugs that are used to treat RA and patient preferences may influence adherence to and outcomes of therapy.

Objectives: The objective of this study was to assess the correlation between the fulfillment of patient preferences and clinical and patient reported outcomes. Methods: PANORAMA was a non-interventional, prospective, multicenter, cohort study. Patients were either biologic naïve or experienced who initiated/switched to anti-TNF at enrollment. Post physician's anti-TNF choice, patients completed a preferences questionnaire over attributes related to anti-TNF treatment. Satisfaction with treatment was assessed with the TSQM questionnaire and compliance (proportion of full doses/planned) was recorded via the use of a patient diary. Persistence was defined as the time period between first and last anti-TNF administration. The observational period was 12 months, with study visits every 3 months.

Results: A total of 254 patients were enrolled in the study. The mean patient age was $58.3 \pm 13.4$ years, $82.7 \%$ were female, $65.4 \%$ were biologic naïve and $66.1 \%$ had severe disease (DAS-28 ESR $>5.1$ ). The mean DAS-28 and HAQ-DI scores at enrollment were $5.5 \pm 1.1$ and $1.4 \pm 0.6$ respectively, while mean disease duration was $6.7 \pm 6.2$ years with $53.2 \%$ of patients being seropositive (RF $(+): 49.2 \%$, Anti-CCP (+): $40.5 \%)$. A monthly administration was most preferred by patients ( $65.7 \%$ vs. $20.1 \%$ for twice per month, $11.8 \%$ for once per week and $3.9 \%$ for twice per week), and the large majority of patients $(75.2 \%)$ preferred the subcutaneous mode of administration. The mean compliance and 12-month persistence rates were $97.0 \%$ and $72.3 \%$ respectively. At 12 months, good EULAR response rate was achieved by $56.5 \%$ of patients and $40.8 \%$ were in DAS- 28 remission. Univariate analysis demonstrated that fulfillment of patient preferences was correlated to good EULAR response $(p<0.001)$, increased probability of being persistent $(\mathrm{p}=0.019)$ and satisfaction with treatment $(\mathrm{p}=0.063)$. Multivariate logistic regression analysis revealed that a good EULAR response was associated with satisfaction of patient preferences (OR 5.560, $p<0.001$ ), good patient knowledge of the disease (OR 1.327, $\mathrm{p}=0.006$ ), absence of history of comorbidities (OR 2.42, $p=0.014$ ) and lower SJC (OR 1.10, $p=0.021$ ), whereas anti-TNF persistence at 12 months was associated (Cox regression analysis) with seropositivity (HR $0.566, p=0.047)$ and a high baseline ESR $(>35 \mathrm{~mm} / \mathrm{h}(\mathrm{HR} 0.587, \mathrm{p}=0.071)$ ).

Conclusions: In anti-TNF treated RA patients, fulfillment of expressed treatment preferences was independently associated with a good EULAR response and correlated with drug persistence at 12 months, emphasizing the importance of patient preferences in treatment outcomes.

Acknowledgements: The study was funded by Merck Sharp \& Dhome S.A., Greece

Disclosure of Interest: None declared

DOI: 10.1136/annrheumdis-2017-eular.4920

\section{FRI0206 CORRELATION BETWEEN THE SERUM ETANERCEPT LEVEL AND RESPONSE TO ETANERCEPT TREATMENT IN PATIENTS WITH RHEUMATOID ARTHRITIS}

S.M. Zivojinovic ${ }^{1}$, M. Sefik-Bukilica ${ }^{2}$, N.S. Damjanov ${ }^{3} .{ }^{1}$ Clinical IV; ${ }^{2}$ Laboratory department ${ }^{3} \mathrm{Head}$ of Institute of rheumatology, Institute of Rheumatology, Belgrade, Serbia

Background: It is well documented that the blockade of TNF- $\alpha$ significantly reduces disease activity in patients with rheumatoid arthritis (RA). However, at least one third of patients receiving etanercept either do not respond to treatment, or lose initial responsiveness [1]. Recent findings indicate that lack of clinical response may be related with lowering the serum drug levels.

Objectives: To investigate the relationship between serum etanercept levels and response to etanercept treatment in patients with RA.

Methods: The study population consisted of fifty eight patients with rheumatoid arthritis (RA), all treated with etanercept. Disease activity was assessed according to the 28-joint count Disease Activity Score (DAS28) at baseline and 6 months of 